Case Report

\title{
Successful Conservative Management of Scrotal Edema Resulting from Uncomplicated Peritoneal Fluid Leak
}

Aymun I Mobark*, Jaafar MA Eltahir, Osman-mahir MA Mahir, Hisham M Ali, Maha A Hummieda, Sara E Khalid, Mohammed Mudawi, Elwaleed Elhassan and Hassan Abu-Aisha

Ribat center for Regular Peritoneal Dialysis, Ribat University Hospital, Khartoum, Sudan

\begin{abstract}
Introduction: Peritoneal fluid leaks are frequent in continuous ambulatory peritoneal dialysis (CAPD) patients and may manifest as subcutaneous or genital edema or as apparent ultrafiltration (UF) failure. Genital swelling in CAPD patients is often due to dialysate leak through a small clinically-undetectable inguinal hernia, and may require herniorrhaphy. If imaging studies exclude an associated hernia or patent processus vaginalis, a conservative approach may be adopted.
\end{abstract}

Case report: An 80 year-old bed-ridden male developed gross bilateral scrotal swelling (without cough impulse) and apparent UF failure shortly after initiation of CAPD. Computed tomography peritoneography (CTP) showed bilateral scrotal fluid collections without a hernia or patent processus vaginalis. An attempt to maintain the patient on CAPD by reducing the dialysate volume, scrotal elevation and adopting a supine position was not successful; CAPD was substituted with temporary hemodialysis (HD) for four weeks. Scrotal swelling reversed after one week of CAPD cessation and did not recur when supine $\mathrm{PD}$ with intermittent dry periods was reinstituted a few weeks later. Satisfactory UF could also be easily attained. This was compatible with caudal fluid migration through peritoneal defects being the underlying cause. The patient had been successfully maintained on CAPD for the last 18 months.

Conclusion: Fluid dissection through soft tissues can result in gross genital edema and apparent ultrafiltration failure in CAPD patients. Supine PD, scrotal elevation with intermittent dry periods may be a practical alternative management in the absence of automated dialysis facilities. The severity of dialysate leak does not preclude a satisfactory response to this conservative management.

\footnotetext{
* Corresponding author; Ribat Center for Regular Peritoneal Dialysis, Burri, P.O. Box 55, Khartoum, Sudan

Email: abuyamn@hotmail.com
}

Keywords: peritoneal dialysis, dialysate leak, genital edema, ultrafiltration failure

\section{Introduction}

Continuous ambulatory peritoneal dialysis (CAPD) has been introduced only recently in Sudan with an experience of about three years, while automated peritoneal dialysis (APD) is not yet available. Infection-related complications, in accordance with what is reported in the literature, are the commonest. However, non infectious complications are not uncommon.

Patients treated with either forms of PD have increased intra-abdominal pressure. As a result, abdominal hernias and dialysate leaks are frequent in the PD population. In a report from a single PD center, the overall rates for hernias and leaks were 0.08 and 0.13 per patient per year respectively [1].

An exit site leak usually refers to the appearance of any wetness around the PD catheter identified as dialysate; however, the spectrum of dialysate leaks also includes any dialysate loss from the peritoneal cavity other than via the lumen of the catheter. Early leakage, occurring at or earlier than 30 days after dialysis initiation, most often manifests as a peri-catheter leak. Late leaks occurring more than 30 days after dialysis initiation may present in a more subtle way with subcutaneous swelling and edema, weight gain, peripheral or genital edema, and apparent ultrafiltration failure [2].

Patients who develop genital edema while on PD without an associated inguinal hernia are labeled as having uncomplicated peritoneal leaks. Such leaks may result from migration of the dialysate through small rents in the peritoneal membrane or at the catheter insertion site; in which case surgical obliteration of the defect may or may not be feasible. 
Figure 1: Lateral view of CTP showing the lower level of the contrast within the peritoneal cavity (arrow); there is no communication between the peritoneal cavity and the scrotum or other abdominal wall defect

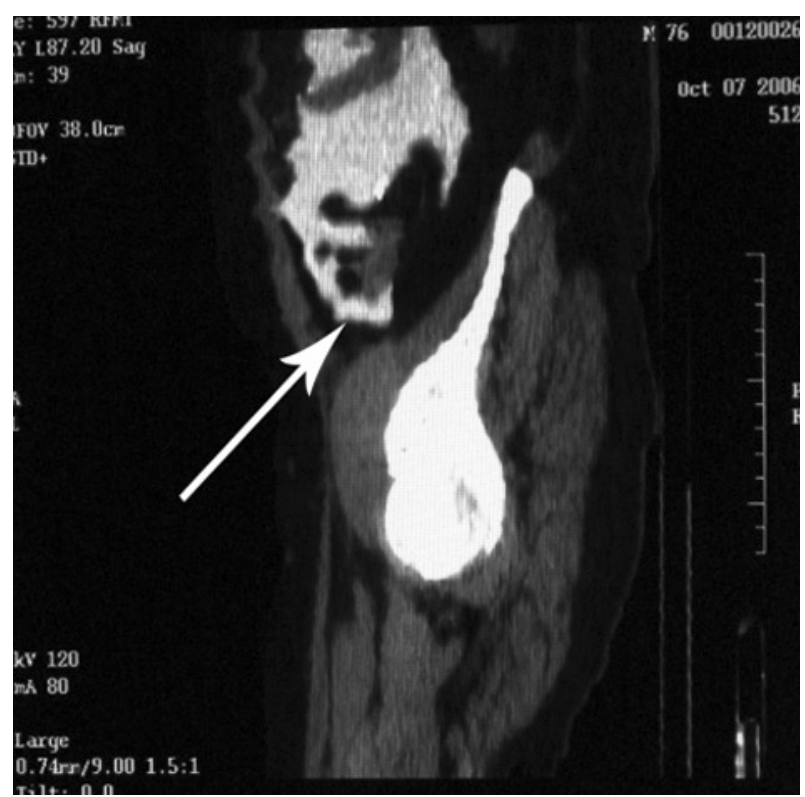

Hence, patients with uncomplicated peritoneal leaks, i.e. without an associated hernia, should initially be treated conservatively with bed rest and temporary hemodialysis (HD) [1]. A short rest period may be sufficient for the tissue defect to heal and allow for the reinstitution of PD.

The case we are presenting here highlights the role of simple conservative management in reversing genital edema complicating PD, in the absence of automated dialysis facilities.

\section{Case report}

An 80 year-old male developed end stage renal disease (ESRD) secondary to hypertensive nephrosclerosis. He also had ischemic heart disease and congestive heart failure with diastolic dysfunction and mitral regurgitation. As a result, he could not tolerate regular HD and was referred for regular PD. In September 2006, a two-cuffed Tenckhoff catheter was inserted using the open surgical technique without complications. The deep cuff was placed within the rectus muscle. Two weeks later, CAPD was commenced with 2-liter exchanges four times per day.

A few days later he complained of painless scrotal swelling of gradual onset. This was associated with a marked reduction in ultrafiltration (UF) volume to less than one liter per day. Examination revealed non tender bilateral scrotal swelling without cough impulse, abdominal wall edema, or clinical evidence of fluid overload. Subsequently, a computed tomography peritoneography (CTP) excluded the presence of coexisting hernia or patent processus vaginalis and a diagnosis of simple fluid leak through the peritoneal membrane (fluid dissection) was made (figures 1-2).

The exchange volume was initially reduced to 1.5 liters then to one liter with adoption of supine position and scrotal elevation for five days without significant improvement. CAPD was then discontinued and the patient temporally transferred to HD. Complete regression of the genital swelling was observed within one week of PD cessation.

Four weeks later, PD was resumed utilizing small dialysate volumes while maintaining the supine position and elevating the scrotum during dwell time. Ambulation and exercise were allowed in gradually increasing

Figure 2: CT peritoneography showing the lower level of the contrast in the pouch of Douglas (picture on the left); no contrast is detectable on distal views (picture on the right).
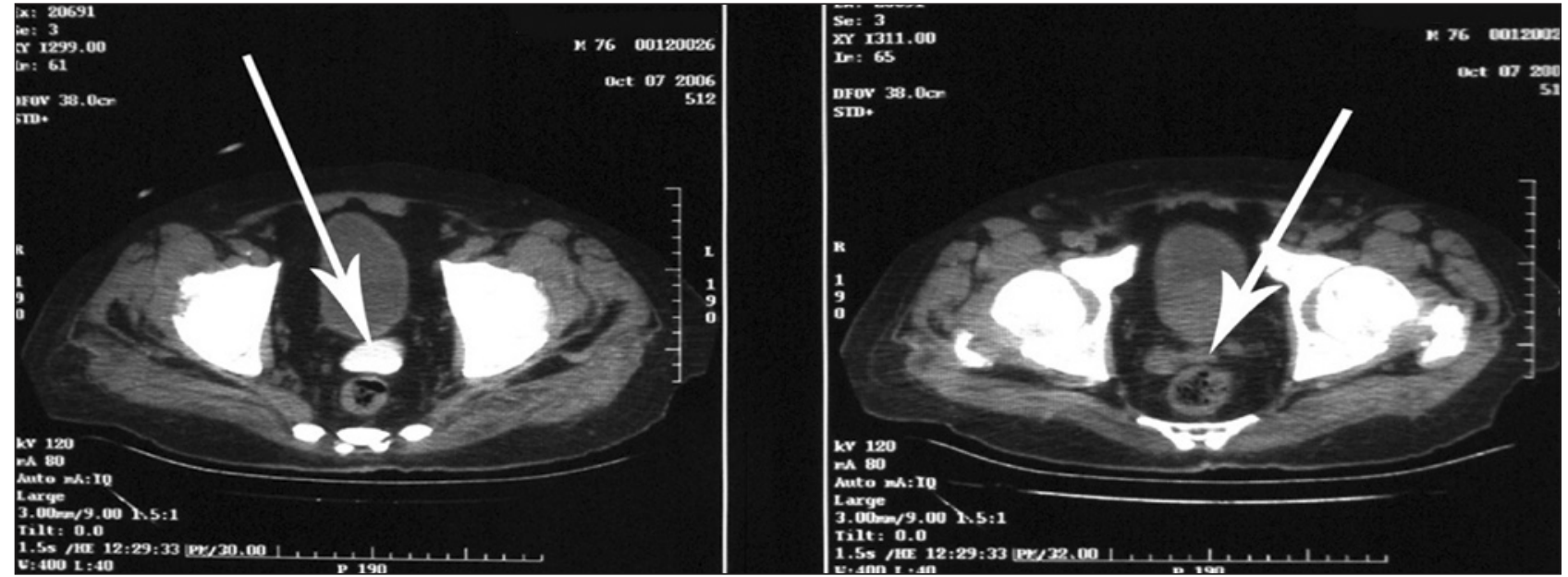

Arab Journal of Nephrology and Transplantation 
increments. Dialysate volumes were increased over one month to two liters per exchange. The scrotal swelling did not recur and a satisfactory ultrafiltration of 1.8 to 2 liters per day could be easily attained. To the time of writing this report, the patient has been maintained on CAPD for 18 months without recurrence of the genital swelling.

\section{Discussion}

Abdominal hernias and peritoneal leaks occur frequently in the PD population. Among a group of 142 unselected patients treated with $\mathrm{PD}$ in a single center, 53 patients (37\%) developed hernia and/or leak during a five-year period. A total of 39 hernias and 63 leaks were registered. Among patients who had hernias, 25 patients $(66 \%)$ required surgical repair of the hernia, with recurrence in seven patients (28\%). Among patients who had peritoneal leaks, 27 patients $(84 \%)$ were initially treated with temporary transfer to $\mathrm{HD}$, low volume APD, or intermittent PD for 4 weeks. The leak recurred in half of the cases and surgical repair was necessary in 12 patients [1].

In an analysis of risk factors associated with abdominal wall complications in PD patients, both hernias and leaks tended to be more common during CAPD compared to APD, but the difference did not reach statistical significance. Polycystic kidney disease was the only factor significantly associated with higher rate of hernias, whereas increased age and higher body mass index were significantly associated with the appearance of leaks. The development of hernia and/or leak did not correlate with the largest peritoneal exchange volume used [1].

Peritoneal leaks in any form can be quite distressing to the patient, and may result in technique failure. Every effort must be taken to minimize their occurrence. The role of surgery in treating dialysate leaks presenting as genital swelling was first highlighted by Cooper et al [3]. He noted that genital edema occurring in patients receiving CAPD is often a sign of a small inguinal hernia which is not detectable clinically, and that the best course of treatment would be early herniorrhaphy.

A patent processus vaginalis is often the underlying pathology in such cases. In one report, this anomaly was found in $12 \%$ of adults who underwent laparoscopic surgery for different indications. After a mean follow up of 5.5 years, $12 \%$ of patients with patent processus vaginalis subsequently required hernia repairs compared to $3 \%$ of patients with obliterated processus vaginalis [4].

Leakage of dialysate into a patent processus vaginalis can result in the formation of a small indirect inguinal hernia which manifests clinically as genital edema without a cough impulse. The correlation of peritoneal fluid leak with this anomaly or other structural defect of the anterior abdominal wall calls for surgical correction. Fluid dissection through the tunica vaginalis manifesting as genital edema, on the other hand, can be treated conservatively [1].

With the advent of peritoneal scintigraphy, computed tomography peritoneography (CTP) and magnetic resonance peritoneography (MRP), a patent processus vaginalis can be reliably identified as the cause of genital edema in PD before surgery is embarked on $[5,6]$. The use of magnetic resonance imaging (MRI) in patients with kidney failure is better avoided because of the recent implication of gadolinium, a contrast agent that is primarily administered during MRI, in the causation of nephrogenic systemic fibrosis [7].

Recent recommendations propose a standard approach to the treatment of both early and late dialysate leaks: 1-2 weeks of rest from CAPD, and surgery in case of recurrence. The outcomes of conservative management are variable. In one study, patients with uncomplicated peritoneal leaks, i.e. no associated hernia, who were initially treated with either low volume supine PD with a dry day or HD for four weeks had a $52 \%$ risk of relapse. However, repeating this technique for an additional four weeks resulted in resolution of the leak in $86 \%$ of the remaining patients [1].

The patient we are reporting had marked dialysate leak manifesting as both genital edema and apparent UF failure. In spite of that, no identifiable anatomical defect could be detected on CTP. This encouraged us to adopt a conservative approach to management, which fortunately succeeded in reversing the problem and allowed the patient to be reinstituted on CAPD.

\section{Conclusion}

Small peritoneal defects undetectable on CTP can result in gross genital edema and apparent UF failure in CAPD patients. Supine PD, scrotal elevation with intermittent dry periods may be a practical alternative management for uncomplicated scrotal edema in the absence of automated dialysis facilities. The severity of dialysate leak does not preclude a satisfactory response to conservative management, which may allow CAPD to be reinstituted after a short rest period.

\section{References}

1. Del Peso G, Bajo MA, Costero O, Hevia C, Gil F, Díaz C, Aguilera A, Selgas R. Risk factors for abdominal wall complications in peritoneal dialysis patients. Perit Dial Int. 2003;23:249.

2. Leblanc M, Ouimet D, Pichette V. Dialysate leaks in peritoneal dialysis. Semin Dial. 2001;14:50. 
3. Cooper JC, Nicholls AJ, Simms JM, Platts MM, Brown CB, Johnson AG. Genital edema in patients treated by continuous ambulatory peritoneal dialysis: an unusual presentation of inguinal hernia. British Medical Journal. 1983;286:1923-24.

4. Van Veen RN, van Wessem KJ, Halm JA, Simons MP, Plaisier PW, Jeekel J, Lange JF. Patent processus vaginalis in the adult as a risk factor for the occurrence of indirect inguinal hernia. Surg Endosc.2007 Feb;21(2):202-5.

5. Hawkins SP, Homer JA, Murray BB, Voss DM, van der Merw WM. Modified computed tomography peritoneography: clinical utility in continuous ambulatory peritoneal dialysis patients. Australas Radio. 2000 Nov;44(4):398-403.

6. Litherland J, Lupton EW, Ackrill PA, Venning M, Sambrook P, Computed tomographic peritoneography: CT manifestations in the investigation of leaks and abnormal collections in patients on CAPD. Nephrol Dial Transplant. 1994;9(10):1449-52.

7. High WA, Ayers RA, Chandler J, Zito G, Cowper SE. Gadolinium is detectable within the tissue of patients with nephrogenic systemic fibrosis. J Am Acad Dermatol 2007; 56:21. 